\title{
Articulatory organization in digit perception and recall
}

\author{
CAROL BERGFELD MILLS and JAMES G. MARTIN \\ University of Maryland, College Park, Maryland 20742
}

\begin{abstract}
In two experiments, tape-recorded strings of seven recall digits were preceded by a redundant stimulus prefix containing either one or three digits. The prefix and recall digits (1) were articulatorily continuous, or (2) were made discontinuous by tape-splicing, or (3) were made discontinuous by deliberately spoken prosodic contour. Subjects were instructed to ignore the redundant prefixes and report only the recall digits. The main results were: (1) Compared to strings preceded by no prefix, all prefix conditions reduced recall. (2) Three-digit prefixes reduced recall less than one-digit prefixes. (3) In most cases, spliced prefixes reduced recall less than intact prefixes. Acoustic waveform measurements are presented to provide a partial account for the results.
\end{abstract}

When the stimulus is a string of seven recall digits preceded by a redundant prefix ("zero") and the required response is only the recall digits, recall is suppressed compared to when the stimulus is only the string of seven digits. This phenomenon is known as the "stimulus prefix effect" (Dallett, 1964, 1965). In later work using this experimental paradigm, Neisser, Hoening, and Goldstein (1969) simply recorded the prefix in a different voice from the voice speaking the recall digits and found that the prefix effect was reduced. Martin (1972) and Mills and Martin (1974) interpreted the Neisser et al. result in terms of the intact articulatory, hence acoustic, organization of the continuous speech stimulus and of the consequences of such organization for ongoing perception. On that view, when a single speaker pronounces the prefix and the recall digits as a continuous utterance, the result is an intact, internally organized acoustic stimulus containing not seven but eight digits. The acoustic organization is compelling and the listener responds to it as such, which means that he or she must accept an articulatorily intact eight-digit string as input to the perceptual process and somehow suppress the prefix before reporting the seven recall digits. However, if one speaker produces the prefix

This work was supported by a grant from the National Institute of Mental Health (MH 16726) and by grants from the Bio-Medical Sciences Support Committee, University of Maryland, to the Center for Language and Cognition. Computing services were provided by the Computer Science Center of the University of Maryland. These experiments were completed while the first author was supported by Training Grant MH 12376 from the National Institute of Mental Health, U.S. Public Health Service. Data from Experiment 1 were reported at the American Psychological Association Convention, Chicago, August 1975. Data from Experiment 2 were reported at the American Psychological Association Convention, Washington, D.C., August 1976. Requests for reprints should be sent to Carol Bergfeld Mills, Psychology Department, University of Maryland, College Park, Maryland, 20742. and another speaker produces the recall digits, obviously there cannot be a single intact stimulus, so the listener should find it easier to separate the prefix from the digit string, as Neisser et al. showed.

Mills and Martin (1974) reported further support for the interpretation that part of the prefix effect is based on the articulatory-acoustic organization of the stimulus. In their experiment, there were two prefix conditions and a no-prefix condition, which are relevant here. In one prefix condition, a speaker pronounced the prefix and recall digits as usual, at a 2 -digit/sec rate. Hence the prefix plus recall digits constituted one intact articulatory unit. Following Dallett's (1964) notation, this was the 07:7 condition, with the colon separating the input stimulus and the required response. In the other prefix condition, the same speaker pronounced the prefix and the recall digits, but the prefix was spliced into the tape prior to the recall string; the prefix had previously been cut out of another tape-recorded utterance spoken by the (same) speaker. In this condition (called the 0/7:7 condition, with the slash indicating the splice), there was therefore no articulatory continuity between the prefix and the following recall digits. The seven recall digits were themselves an intact articulatory unit, in fact a tape copy of the same seven-digit string spoken without a prefix, although in this condition they were preceded by a spliced-on prefix. The results in the experiment showed that the (spliced) $0 / 7: 7$ strings were recalled more easily than the (unspliced, hence intact) 07:7 strings, though not as easily as the same seven-digit strings not preceded by a prefix. These results support the view that a part of the prefix effect (poorer recall) can be accounted for by the articulatory continuity of the prefix and recall string.

It is worth noting that the Mills and Martin (1974) results are at least mildly surprising. On their view of articulatory organization, any experimental dis- 
tortions of the natural acoustic speech stimulus, particularly those resulting in articulatorily impossible sequences, increase processing demands (Martin, 1975), although the "cost"' in processing is greater for some distortions than for others. The acoustic effects of tape splicing in the experiment were subtle; yet they produced differences in recall, a very coarse response measure. Many investigators have used tape-splicing manipulations (e.g., Abrams \& Bever, 1969; Wingfield \& Klein, 1971), but typically those investigators were concerned with response measures other than memory. However, Wingfield and Klein (1971) also measured sentence recall in their study and reported lower scores for spliced than for unspliced sentences (see also Darwin, 1975). Both Mills and Martin's (1974) and Wingfield and Klein's (1971) results show that the subtle distortions of the acoustic input caused by splicing have perceptual consequences, although splicing has opposite effects on recall in terms of the differing task requirements in the two experiments.

The present paper further examined the effects of articulatory continuity on the stimulus prefix effect in two ways. First, additional experimental conditions were used. Second, listener impression and acoustic waveform measurements were obtained to attempt to provide a more detailed explanation for the experimental results.

In one condition of the Neisser et al. (1969) experiment, a three-digit prefix (three "zeroes") rather than a single-digit prefix spoken by a single speaker preceded the recall digits. The results were that, unlike the singledigit prefix condition, the three-digit prefix did not reduce recall. These results can be explained by the articulatory continuity hypothesis by assuming that the speaker set the three-digit prefix apart from the recall digits by intonation contour or other prosodic cue, however slight. Another possibility (hereafter, the grouping hypothesis) is that the three successive "zeroes" in a three-digit prefix form a "group" based on gestalt-like notions such as (physical) similarity or (conceptual) identity, and that they can be more easily separated perceptually from the recall digits for that reason. This grouping hypothesis was approximately the one suggested by Neisser et al. (1969). A third possibility, also plausible, is that the three-digit prefix allowed the listener more time to get set to suppress or ignore the prefix sequence and attend to the subsequent recall digits (the time-for-set hypothesis).

In summary, the articulatory hypothesis focuses upon the rate of change of physical events over time, that is, the time-varying spectrum of the natural speech stimulus (Jones, 1976; Meltzer, Martin, Mills, Imhoff, \& Zohar, 1976). In contrast, a grouping hypothesis rests solely upon the similarity or identity of events regardless of timing, while a set hypothesis identifies only time, not the concommitant events, as the crucial variable. These differences lead to the expectation that disturbances in rate of change between prefix and subsequent events (e.g., continuity vs. discontinuity) will account for the prefix effect under the articulatory hypothesis regardless of the length of the prefix. On the other hand, under either the grouping or set hypothesis, longer strings of identical events should be more readily separated and ignored as prefixes than shorter ones, regardless of acoustic continuities between prefix and subsequent items. These alternative hypotheses were considered in Experiment 1.

\section{EXPERIMENT 1}

Recall strings were preceded by a three-digit prefix in three different conditions: (1) the prefix and recall digits were articulatorily continuous (intact); (2) they were made discontinuous by splicing; (3) they were made discontinuous by deliberately spoken prosodic contour. On the articulatory hypothesis, when the prefix and recall digits were separated either by the spoken contour or by splicing, recall should be better than when the prefix and recall digits were spoken continuously. On the grouping hypothesis or the time-for-set hypothesis, recall should be the same in all three of the three-digit prefix conditions. Two one-digit prefix conditions and one no-prefix condition provided additional comparisons. The six conditions are shown in Table 1 . In the present experiment, one procedural change was made. While Mills and Martin (1974) and Neisser et al. (1969) used "zeroes" as prefix digits, the present experiments used "ones."

\section{Method}

Materials. The 72 strings of seven digits were random permutations, with the restriction that no more than two consecutive digits in either ascending or descending order were included. The twosyllable digits "seven" and "zero" were not used, nor was "one" because it was used as the prefix.

The digit strings were read into a tape recorder by a male speaker at the rate of 2 digits/sec in time with a metronome. All strings were preceded by a spoken "ready" $1 \mathrm{sec}$ before the prefix (if present) or the first recall digit (if there was no prefix).

Table 1

Experimental Conditions

\begin{tabular}{llcc}
\hline & \multicolumn{3}{c}{ Prefix } \\
\cline { 2 - 4 } $\begin{array}{l}\text { Relation to Recall } \\
\text { String }\end{array}$ & $\begin{array}{c}\text { None } \\
\text { () }\end{array}$ & $\begin{array}{c}\text { One Digit } \\
(1)\end{array}$ & $\begin{array}{c}\text { Three Digits } \\
(111)\end{array}$ \\
\hline $\begin{array}{l}\text { Control } \\
\text { Intact } \\
\text { Continuous }\end{array}$ & $7: 7$ & & $1117: 7$ \\
$\begin{array}{l}\text { Speaker } \\
\text { Discontinuous }\end{array}$ & $17: 7$ & $111,7: 7$ \\
$\begin{array}{l}\text { Spliced } \\
\text { Discontinuous }\end{array}$ & & $111 / 7: 7$ \\
\hline
\end{tabular}


A master recording was made of each of the 72 strings, each spoken four times consecutively to produce four prefix conditions: (1) no prefix (7:7), (2) single-digit prefix (17:7), (3) three-digit prefix with intonation contour $(111,7: 7)$, and (4) three-digit prefix without contour (1117:7). Each string was spoken in the four ways consecutively to keep intensity, etc., as close to identical as possible, and to help to ensure smooth speech flow in the spliced conditions where part of one copy of a string was spliced onto another copy of the same string. As in earlier experiments (Mills \& Martin, 1974), digit timing on the first channel was controlled by feeding metronome beats into the second channel. As the beats were recorded, they were monitored through headphones by the speaker who spoke the digit strings in time to the beats. The metronome beats were not audible on the channel on which the digits were recorded.

Tape copies were used to construct the strings in the spliced (1/7:7 and 111/7:7) conditions. To construct a spliced string, the "ready" and prefix were removed from a copy of a string with a prefix, and were spliced onto the matched no-prefix copy of the same string from which the "ready" had been removed.

Precise timing of the prefix and the first recall digit in the spliced (1/7:7 and 111/7:7) strings was facilitated by the recorded metronome beats on the second channel, as in the earlier study (see Mills \& Martin, 1974). (The results of these manipulations as they appeared on an acoustic waveform display, together with listener impressions will be discussed later.)

Six parallel counterbalanced tapes were constructed such that a given string appeared on each tape in a different experimental condition. Each tape consisted of six blocks of 12 strings: one no-prefix block, two blocks with a one-digit prefix, and three blocks with a three-digit prefix. All of the strings in the noprefix block were 7:7 strings. Half of the strings in each block with a one-digit prefix were 17:7 strings and half were 1/7:7 strings. One-third of the strings in the remaining three blocks were in the $1117: 7,111 / 7: 7$, and $111,7: 7$ conditions.

Procedure. First, 12 eight-digit practice strings were presented auditorily to individual subjects by tape recorder through headphones. A subject was instructed to listen to each string of digits and to recall the string orally immediately after hearing it, while pointing to corresponding boxes in a grid of eight boxes to indicate the serial position of the digits. The subject was told to say "blank" if he or she could not remember a particular digit.

For the experimental strings, the subject was given a grid of seven boxes and was instructed to point to the boxes during recall as before. Responses were tape-recorded and simultaneously written down by the experimenter. Each subject heard one of six experimental tapes. Blocks containing the same conditions were never heard consecutively. Prior to listening to prefix blocks, the subject was told to ignore the "one" (or "ones") and just recall the following seven digits. Prior to listening to the no-prefix block, the subject was instructed to recall the seven digits. The subject was given approximately $20 \mathrm{sec}$ in which to recall each string.

Subjects. The subjects were 108 extra-credit volunteers from introductory psychology classes. There were 18 subjects per counterbalanced tape, with three subjects hearing a given tape in each of six starting orders.

\section{Results}

Two measures of recall were calculated: (1) the number of completely correct strings, and (2) thenumber of digits recalled in their correct serial position. A string was considered completely correct if all and only seven digits were recalled in their apropriate serial position. In scoring digits correct, only the first seven digits reported were considered, and a digit was counted as correct only if recalled in the appropriate absolute serial position.

All analyses of variance included Experimenter
Table 2

Mean Strings and Digits Correctly Recalled in Experiment 1

\begin{tabular}{lllllll}
\hline & \multicolumn{6}{c}{ Condition } \\
\cline { 2 - 7 } & $7: 7$ & $111,7: 7$ & $111 / 7: 7$ & $1117: 7$ & $1 / 7: 7$ & $17: 7$ \\
\hline & \multicolumn{6}{c}{ Strings } \\
Mean & 7.79 & 7.17 & 7.12 & 6.43 & 6.19 & 5.30 \\
Percent & 65 & 60 & 59 & 54 & 52 & 44 \\
& \multicolumn{7}{c}{ Digits } \\
Mean & 72.95 & 70.96 & 70.59 & 68.20 & 66.89 & 62.50 \\
Percent & 87 & 84 & 84 & 81 & 80 & 74 \\
\hline
\end{tabular}

Note-For strings, maximum score $=12$; for digits, maximum score $=84$.

and Tape as between-subjects factors: these are ignored below. Newman-Keuls tests were applied to all reported paired comparisons of experimental conditions.

Strings correct. The mean number and percent of completely correct strings recalled in the six conditions are shown in Table 2. Differences among conditions were significant, $F(5,450)=32.93, p<.001$, as were differences between pairs of conditions $(p<$ .01 ), except those between the 111,7:7 and the 111/7:7 conditions and between the $1117: 7$ and $1 / 7: 7$ conditions.

Digits correct. Differences among conditions using this measure are also shown in Table 2 and were significant, $F(5,450)=44.74, p<.001$, as was Serial Position, $F(6,540)=1,038.67, p<.001$, and the interaction between Condition and Serial Position, $F(30,2700)=7.94, p<.001$. The form of this interaction can be seen in Figure 1. Notice that there is very little difference between conditions at the beginning and end of the serial position curves. All differences between pairs of conditions were significant $(p<.01)$ except between the $111 / 7: 7$ and the $111,7: 7$ conditions.

Summarizing the results of Experiment 1: (1) Recall

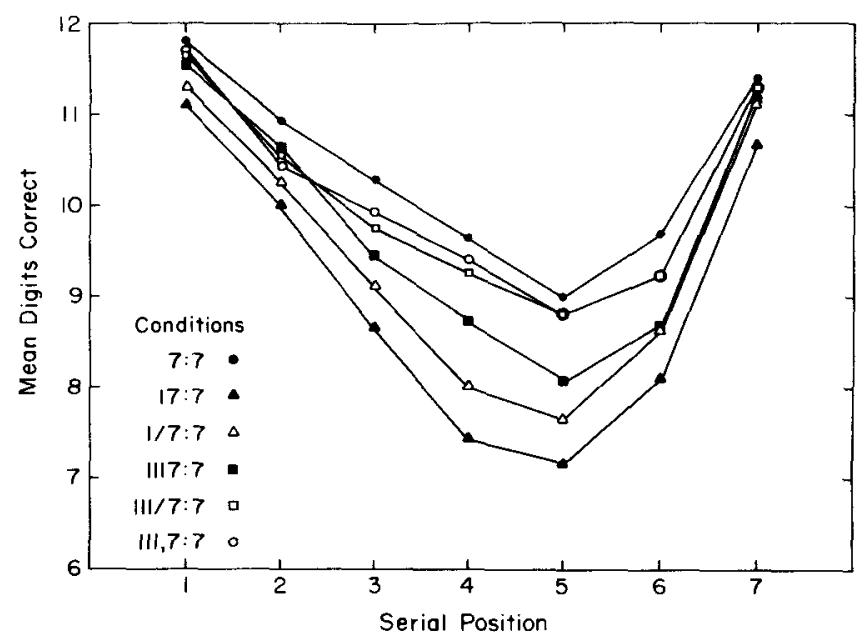

Figure 1. Mean number of digits correct in each serial position. 
was reduced in all prefix conditions compared to the no-prefix condition. This result differs from that of Neisser et al. (1969), who found no recall reduction with a three-digit prefix. The difference between their results and ours is probably due to our use of "ones" rather than "zeroes." "Zeroes" seem intuitively easier to separate perceptually during recall for two reasons: "Conceptually" they are less like the recall digits, and phonetically they contain two syllables, unlike all recall digits except "seven" (used by Neisser et al. but not by us). (2) Spliced one- or three-digit prefixes reduced recall less than their intact counterparts, as expected on the articulatory continuity hypothesis, although the differences were smaller between conditions with three-digit prefixes than with one-digit prefixes. (3) In general, three-digit prefixes reduced recall less than did one-digit prefixes. Hence, even though the results in the conditions with three-digit prefixes support the articulatory organization hypothesis, grouping and time-for-set factors cannot be completely ruled out. (4) By far the largest stimulus prefix effect (poorest recall) occurred in the intact one-digit-prefix condition (17:7). To further investigate this finding, Experiment 2 was conducted.

\section{EXPERIMENT 2}

In Experiment 1, three-digit prefixes reduced recall less than one-digit prefixes. There are at least two possible explanations for this result. (1) Any effects of articulatory discontinuity (111/7:7 condition) are minor compared to those provided by the opportunity for grouping or time-for-set when a three-digit prefix is used. Put another way, in the case of onedigit prefixes, articulatory discontinuity (1/7:7 condition) may be particularly effective for reducing the magnitude of the prefix effect simply because the opportunities for grouping or time-for-set are not available to the listener when the prefix is only one digit. (2) The effetts of articulatory continuity as such are greater in the single-prefix case because of differences in the way intact one-prefix vs. threedigit-prefix strings are spoken. Experiment 2 was done to consider these possibilities.

Three conditions in Experiment 2 used a three-digit prefix. In one, splices were placed between the digits making up the three-digit prefix, but the third digit of the prefix was an intact part of a one-digit prefix and recall string, that is, a $1 / 1 / 17: 7$ condition. Since the first and second digits of the prefix were tape copies of the third prefix, the three prefix digits were not merely similar, they were acoustically identical. Hence grouping and time-for-set effects are as available to the listener in this condition as in other threedigit-prefix conditions. Recall of these strings was compared with that of the strings in the three-digitprefix spliced (111/7:7) and intact (1117:7) condi- tions as used in Experiment 1. The single-digit intact (17:7) condition was also included. On the articulatorily based hypothesis that the relatively larger reductions in recall following a one-digit prefix compared to a three-digit prefix are based on differences in the way the respective intact strings are pronounced, recall results, from best to worst, should occur in the order 111/7:7, 1117:7, 1/1/17:7, 17:7.

\section{Method}

This experiment differed from Experiment 1 in the following ways. In conditions $17: 7,1117: 7$, and $111 / 7: 7$, the stimulus strings were those used in Experiment 1. The strings for the 1/1/17:7 condition were constructed from three copies of the 17:7 strings used in Experiment 1 . They were constructed by splicing copies of the same prefix "one" onto the original 17:7 string so that, when completed, the three prefixes were acoustically identical, although the last of the three was continuous with the following digits, hence the $1 / 1 / 17: 7$ notation.

The three kinds of three-digit-prefix strings were combined into blocks, as in Experiment 1, except that there were three blocks of 18 strings each. These blocks and the one block of 17:7 strings were counterbalanced as in Experiment 1. There were 112 subjects divided evenly into groups hearing each of four experimental tapes each in four starting orders.

\section{Results}

Strings correct. Table 3 shows the results. The differences between conditions were significant, $F(3,288)=42.46, p<.001$. All differences between pairs of conditions were significant (ps $<.05)$ except the comparison between the 111/7:7 and the 1117:7 conditions.

Digits correct. The following effects were significant: Condition, $F(3,288)=49.03, p<.001$, Serial Position, $F(6,576)=187.84, p<.001$, and the interaction between Condition and Serial Position, $F(18,1728)=8.4, p<.001$. The form of this interaction was similar to that in Experiment 1 . All differences between pairs of conditions were significant $(p<.01)$ except that between the 1117:7 and 111/7:7 conditions.

In summary, recall scores between spliced (111/7:7) and intact (1117:7) three-digit-prefix strings did not differ in Experiment 2, but they were better than for

Table 3

Mean Strings and Digits Correctly Recalled in Experiment 2

\begin{tabular}{lcccc}
\hline & \multicolumn{4}{c}{ Condition } \\
\cline { 2 - 5 } & $111 / 7: 7$ & $1117: 7$ & $1 / 1 / 17: 7$ & $17: 7$ \\
\hline \multicolumn{5}{c}{ Strings } \\
Mean & 11.01 & 10.88 & 10.28 & 8.17 \\
Percent & 61 & 60 & 57 & 45 \\
& \multicolumn{4}{c}{ Digits } \\
Mean & 106.85 & 106.69 & 103.38 & 95.81 \\
Percent & 85 & 85 & 82 & 76 \\
\hline
\end{tabular}

Note-For strings, maximum possible $=18$; for digits, maximum possible $=126$. 
recall of $1 / 1 / 17: 7$ strings, which in turn faired better than 17:7 strings. Hence, these results suggest that part of the reason for the relatively large recall reductions in an intact one-digit-prefix string, compared to an intact three-digit-prefix string, were based on subtle pronounciation, hence acoustic, differences. To help in explaining these experimental results, further data were obtained on the perceptual and acoustic consequences of splicing and other experimental manipulations.

\section{LISTENER IMPRESSIONS AND ACOUSTIC WAVEFORM MEASUREMENTS}

\section{Listener Impressions}

Four research assistants independently listened twice to each prefix string used in Experiment 1 and attempted to identify its condition (e.g., spliced or not). Measure 1 of Table 4 shows the results of their second listening session. Errors are shown for each listener to indicate interjudge variability. Note that listener T.B., who spoke the digit strings, did not have the lowest error rate. As can be seen, the error tendency was highest for spliced three-digit-prefix $(111 / 7: 7)$ strings. When these strings were misjudged, $87 \%$ of the time they were reported as unspliced $(1117: 7)$ rather than contoured $(111,7: 7)$.

Three judges listened to all strings a third time and judged whether the first digit following the prefix was heard as "early," "on time," or "late," based upon the rhythmic context provided by the prefix. As Table 4, Measure 2 shows, intact (17:7 or 1117:7) strings were reported as on time. Digits separated from the prefix by deliberate prosodic contour (111, $7: 7)$ were reported as late. When the digits were spliced $(1 / 7: 7$ or $111 / 7: 7)$, the tendency to rate them late was somewhat stronger.

\section{Waveform Measurements}

The digitized acoustic waveform of each string was displayed on a PDP-12 computer screen, and time between offset of last digit of the prefix and onset of first recall digit was recorded (background noise reduced the accuracy of these measurements to an unknown extent). As Table 4, Measure 3 shows, there was little silence between the prefix and the recall digits in the case of intact (17:7 and 1117:7) strings; medians were $00 \mathrm{msec}$ because nearly $60 \%$ of these strings had no silence between the prefix and recall digit. Spliced (1/7:7 and 111/7:7) strings had larger intervals of silence between the prefix and recall digit. These results are in line with the listener impressions shown in Measure 2 of Table 4 that spliced recall digits tended to be "late."

One possible reason for this result might be substantial error in the splicing manipulations. This possibility was ruled out by measurements of the intervals between timing clicks on the second channel.
Table 4

Listener Impressions and Waveform Measurements

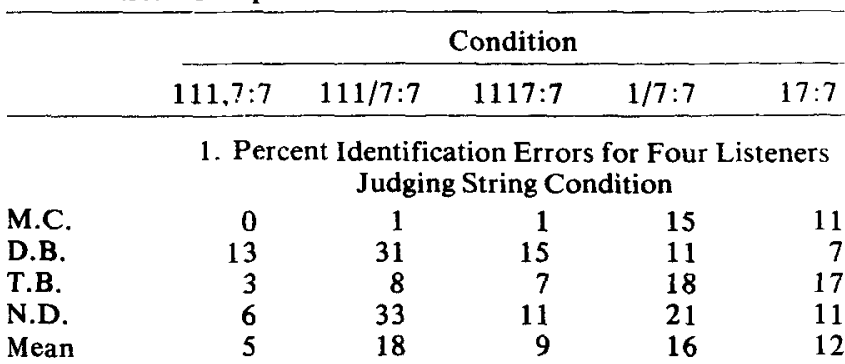

2. Percent Timing Judgments Averaged Over Three Listeners

\begin{tabular}{lrrrrr} 
Early & 1 & 9 & 4 & 19 & 7 \\
On Time & 63 & 37 & 93 & 32 & 89 \\
Late & 36 & 54 & 3 & 49 & 4 \\
\multicolumn{5}{c}{ 3. Silence (milliseconds) Between Prefix } \\
Median & 16 & 72 & 00 & 68 & 00 \\
Mean & 31 & 77 & 16 & 70 & 20 \\
Range & $0-125$ & $0-175$ & $0-100$ & $0-175$ & $0-100$ \\
& \multicolumn{5}{c}{ and Recall Digit } \\
N & \multicolumn{5}{c}{ Silence (milliseconds) Between Digit } \\
Mean & 46 & 69 & 28 & 64 & 31 \\
\hline & 50 & 8 & 46 & -1 & 10 \\
\hline
\end{tabular}

Whether a splice intervened or not, the time between the clicks "under" the last prefix digit and first recall digit averaged about $490 \mathrm{msec}$ (the "expected" value was $500 \mathrm{msec}$ ) for all five conditions, with standard deviations less than $8 \mathrm{msec}$.

Silence between the onset of the first recall digit and its associated click was also recorded for every string in which silence occurred between the prefix and first recall digit. These values indicate the amount of time that digit onset preceded the click and should be generally positive, since speakers tend to align syllabic vowels, not syllable onsets, with timing clicks (Allen, 1972). As Measure 4 of Table 4 shows, in the case of the unspliced conditions in which the recall digits were preceded by a three-digit prefix (1117:7 and 111,7:7), the first recall digits were spoken relatively early in relation to their associated timing clicks, that is, the clicks followed the digit onset at relatively large time intervals. However, the recall digits preceded either by a single prefix $(17: 7)$ or no prefix in their original utterances $(1 / 7: 7$ and $111 / 7: 7$ ) were spoken late, relative to their associated timing clicks, as indicated by the smaller values in Measure 4 of Table 4 . This result might suggest that the speaker started a bit late in uttering the earlier items of a string, whether prefix or recall digits, i.e., that he required several items to get "on the beat."

\section{DISCUSSION}

These and earlier experiments show that a redundant stimulus prefix generally produces greatest 
reductions in digit recall when the prefix and recall digits are articulatorily continuous. While the results in these experiments do not completely rule out grouping or time-for-set effects in the stimulus prefix experiments, they do provide support for articulatory organization effects in the prefix experiments as well as in speech perception in general. In addition, the present experiments suggest that the prefix effect is most persistent when it involves the early items of an intact string. In Experiment 1, the intact one-digit prefix produced greatest interference, while in Experiment 2 the same intact one-digit prefix spliced into a three-digit-prefix string produced greater interference than the other three-digit-prefixes. Some evidence suggests that these results may be due to the fact that early digits in a string are pronounced differently than later digits, in particular, that they are shorter and somewhat more "compressed" than later digits.

Consider the data in Measure 4 of Table 4. Notice first that, compared to all other strings, the first recall digits in the $1117: 7$ and $111,7: 7$ strings were spoken relatively early, as mentioned above. In both conditions, the first recall digit was the fourth digit in a string of 10 digits. On the other hand, the initial recall digits in the other types of strings were either the first digit in their original intact strings $(1 / 7: 7$ and $111 / 7: 7$ conditions) or the second digit in their strings (17:7 condition). This suggests that earlier digits in a string are somewhat "compressed" relative to later digits. Possibly the early digits are in some articulatory sense more "tightly" encoded, in which case it is not surprising that a single prefix is not easily disentangled perceptually from the following recall digits. The same explanation applies to the reduced recall in the $1 / 1 / 17: 7$ condition of $E x-$ periment 2 compared to other three-digit-prefix conditions, since these were constructed from strings in the 17:7 condition of Experiment $1 .^{1}$ Notice that silent time between the prefix and recall digit will not explain this result; these times are the same following the more easily separable 1117:7 strings as following the less easily separable 17:7 strings, as can be seen in Measure 3 of Table 3.

The notion that early digits are "compressed" is not entirely without precedent. There is some evidence that early syllables in an utterance tend to shorten as a function of the number of syllables following (e.g., Oller, 1973). Whatever the reasons for this phenomenon, it seems to be part of the mechanism for speaking.

\section{REFERENCES}

Abrams, K., \& Bever, T. G. Syntactic structure modifies attention during speech perception and recognition. Quarterly Journal of Experimental Psychology, 1969, 21, 280-290.

AlLEN, G. D. The location of rhythmic stress beats in English: An experimental study I. Language and Speech, 1972, 15, 72-100.

DALLETT, K. M. Effects of redundant prefix on immediate recall. Journal of Experimental Psychology, 1964, 67, 296-298.

Dallett, K. M. "Primary memory": The effects of redundancy upon digit repetition. Psychonomic Science, 1965, 3, 237-238.

DARWIN, C. J. On the dynamic use of prosody in speech perception. In A. Cohen \& S. Nooteboom (Eds.), Structure and process in speech perception. New York: Springer-Verlag, 1975.

JoNEs, M. R. Time, our lost dimension: Toward a new theory of perception, attention and memory. Psychological Review, 1976, 83, 323-355.

MarTiN, J. G. Rhythmic (hierarchical) versus serial structure in speech and other behavior. Psychological Review, 1972, 79, 487-509.

MARTIN, J. G. Rhythmic expectancy in continuous speech perception. In A. Cohen \& S. Nooteboom (Eds.), Structure and process in speech perception. New York: Springer-Verlag, 1975.

Meltzer, R. H., Martin, J. G., Mills, C. B., ImHoff, D. L., \& ZOHAR, D. Reaction time to temporally-displaced phoneme targets in continuous speech. Journal of Experimental Psychology: Human Perception and Performance, 1976, 2, 277-290.

Mills, C. B., \& Martin, J. G. Articulatory organization in the prefix effect. Perception \& Psychophysics, 1974, 16, 309-314.

Neisser, U., Hoenig, Y. J., \& Goldstein, E. Perceptual organization in the prefix effect. Journal of Verbal Learning and Verbal Behavior, 1969, 8, 424-429.

OLLER, D. K. The duration of speech segments: The effect of position in utterance and word length. Journal of the Acoustical Society of America, 1973, 54, 1235-1273.

Wing field, A., \& Krein, J. F. Syntactic structure and acoustic pattern in speech perception. Perception \& Psychophysics, 1971, 9. 23-25.

\section{NOTE}

1. As further evidence for this interpretation, it is interesting to note that prefix "intrusions" (i.e., the subject reported the prefix as the first recall digit), although rare, occurred only in the 17:7 and $1 / 1 / 17: 7$ conditions and approximately evenly between the two conditions.

(Received for publication May 12, 1976; revision accepted January 29, 1977.) 